\title{
News on Silicon Drift Detectors for High Resolution EDX Imaging and Spectroscopy
}

Adrian Niculae ${ }^{1}$, Thiago Barros ${ }^{1}$, Alois Bechteler ${ }^{1}$, Robert Lackner ${ }^{1}$, Kathrin Hermenau ${ }^{1}$, Klaus Heizinger $^{2}$, Tristan Mönninghoff ${ }^{1}$, Heike Soltau ${ }^{1}$ and Lothar Strüder ${ }^{2}$

1. PNDetector GmbH, München, Germany.

2. PNSensor GmbH, München, Germany.

* Corresponding author: adrian.niculae@pndetector.de

Introduced into the Energy Dispersive X-ray (EDX) Microanalysis applications more than 15 years ago, Silicon Drift Detectors (SDD) fabricated by PNDetector have established themselves as state-of-the-art detectors in many of the EDX systems used in SEMs and (S)TEMs. Over all these years we have been continuously working on improving the detector spectroscopic performance.

Featuring the monolithically integrated first FET onto the detector chip which insures the lowest achievable total input capacitance and thus the lowest possible noise level, the SDDs fabricated by PNDetector have already reached the theoretical limits in terms of energy resolution and light element sensitivity.

Since 2015 the SDD wafer fabrication takes place in a newly built silicon detector fabrication facility in Munich Neuperlach. This facility is dedicated to sensor manufacturing and allows us a full control of all relevant fabrication processes and therefore of critical detector parameters such as detector leakage current, detection efficiency at light elements and radiation hardness. On the other side it gives us the full manufacturing flexibility in terms of detector types and numbers for the serial production, as well as for testing new designs, new geometries, new features.

With the new fabrication facility, the SDD performance was brought to a new level not only in terms of noise and energy resolution, but also with regard to other features which are of great importance for EDX in SEM/(S)TEM such as light element performance or radiation hardness.

Fig1. shows the typical spectroscopic performance for three representative detector types and sizes. Whereas the SD3 ${ }^{\text {plus }}$ type offers the best energy resolution (Fig. la, green curve) and light element performance (Fig. 1b) due to its special FET and anode design featuring the smallest achievable input capacitance of about $50 \mathrm{fF}$, SDDs of larger sizes, e.g. $100 \mathrm{~mm}^{2}$ also are capable of reaching energy resolution values very close to the physical limits $\left(119 \mathrm{eV}\right.$ for $\mathrm{Mn}-\mathrm{K}_{\mathrm{a}}$ ).

The results of the radiation hardness tests performed on selected devices are shown in Fig. 2 where the energy resolution of the $\mathrm{C}-\mathrm{K}$ and $\mathrm{Mn}-\mathrm{K}_{\mathrm{a}}$ lines is plotted against the cumulated photon dose striking the detector. Fig 2 a demonstrates that the radiation hardness of the detectors from the new facility could be improved by more than a factor of 3 compared to the old facility. The degradation in Carbon resolution is less than $10 \%$ up to almost $10^{13}$ photons, for the $\mathrm{Mn}-\mathrm{K}_{\mathrm{a}}$ line there is no sign of degradation.

Another main stream in our detector development activities is the optimization of both sensor geometry and package configuration with respect to the x-ray collection efficiency (solid angle) from the analyzed sample. This is the case for the well-known pole-piece Rococo2 annular detector configuration (Fig. 3a) which is capable of achieving an unprecedent solid angle of $1.8 \mathrm{sr}$ or the Racetrack detector series (Fig. $3 b)$ which are very successfully used in many (S)TEM EDX systems. 


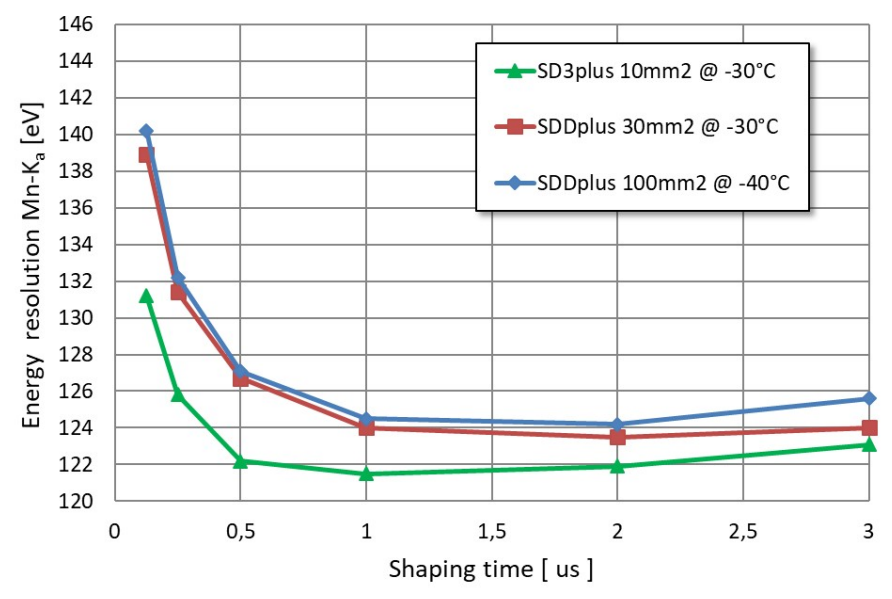

(a)

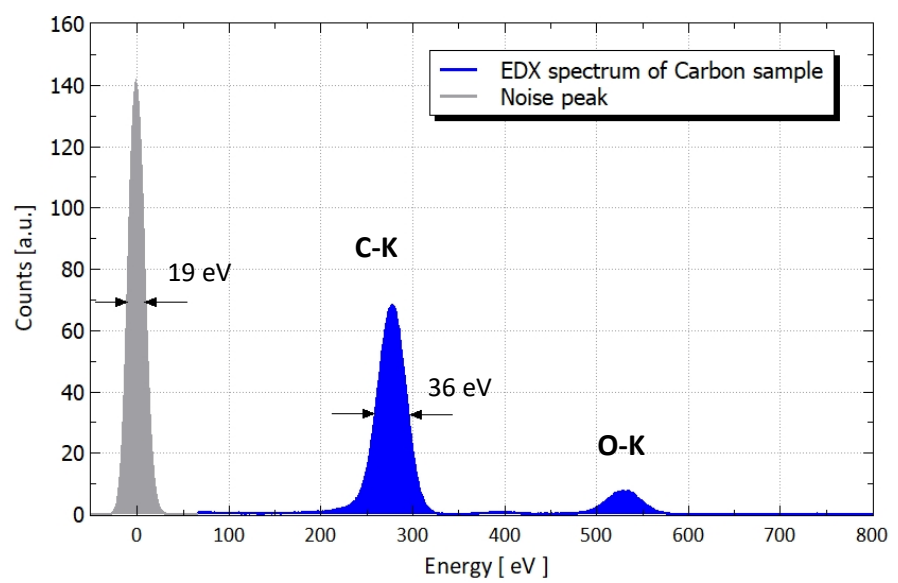

(b)

Figure 1. (a) Energy resolution of various types of SDDs as a function of shaping time;

(b) EDX spectrum of a Carbon sample measured with a $10 \mathrm{~mm}^{2} \mathrm{SD}^{\text {plus }}$ detector.
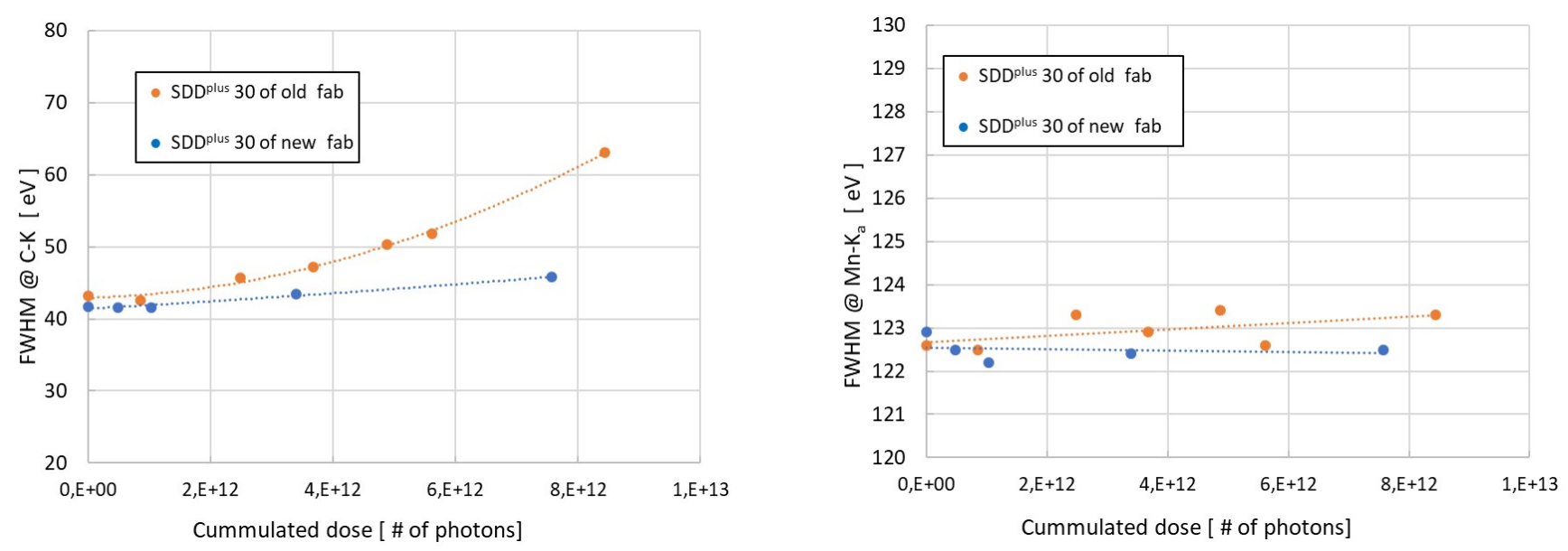

Figure 2. Energy resolution of $30 \mathrm{~mm}^{2} \mathrm{SDD}^{\text {plus }}$ devices measured at Carbon and $\mathrm{Mn}-\mathrm{Ka}$ lines against the cumulated photon dose.

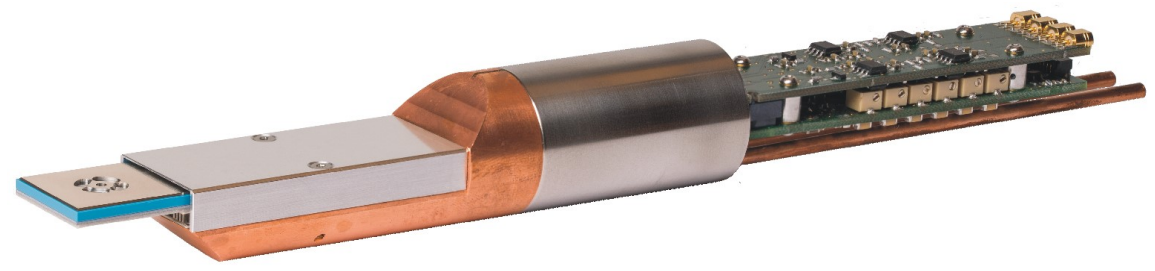

(a)

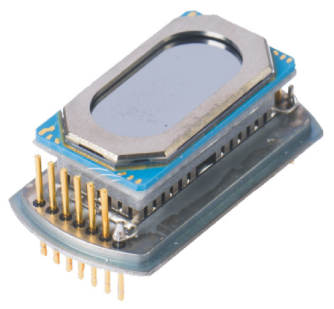

(b)

Figure 3. Special detector geometries like Rococo2 annular detector or Racetrack SDD optimized for maximum $\mathrm{x}$-ray collection efficiency from the samples. 